\title{
Ranking the return on assets of Tehran stock exchange by a new method based on Z-numbers data
}

\section{Maryam Farzam}

Islamic Azad University Tehran North Branch

Mozhdeh Afshar kermani ( $\nabla$ mog_afshar@yahoo.com)

Islamic Azad University Tehran North Branch https://orcid.org/0000-0002-5912-058X

\section{Tofigh Allahviranloo}

Istinye university

\section{Azim Aminataei}

K.N.Toosi University Of Technology

\section{Research Article}

Keywords: Z-numbers, Ranking, Centroid Point, Return on assets, Stock exchange

Posted Date: August 10th, 2022

DOI: https://doi.org/10.21203/rs.3.rs-1017049/v2

License: @ (i) This work is licensed under a Creative Commons Attribution 4.0 International License. Read Full License 


\title{
Ranking the return on assets of Tehran stock exchange by a new method based on Z-numbers data
}

\author{
Maryam Farzam ${ }^{1}$ Mozhdeh Afshar kermani ${ }^{1 *}$ \\ Tofigh Allahviranloo ${ }^{2}$ Azim Aminataei ${ }^{3}$ \\ ${ }^{1}$ Department of Mathematics, Tehran North Branch Islamic Azad University, Tehran, Iran. \\ ${ }^{2}$ Faculty of Engineering and Natural Sciences, Bahcesehir University, Istanbul, Turkey. \\ ${ }^{3}$ Faculty of Mathematics, K.N.Toosi University Of Technology,P.O.Box: 16765-3381, Tehran, Iran.
}

\begin{abstract}
Since real-world data is often inaccurate and working with fuzzy data and Z-numbers are very important and necessary, in the real world we need to rank and compare data. In this paper, we introduce a new method for ranking Z-numbers. This ranking algorithm is based on centroid point.

We evaluate distance between centroid point, and based on this distance, we rank the Z-numbers.

We use this method in two practical examples. First in ranking the return on assets of Tehran stock exchange, and second, in ranking of factors affecting the productivity of tourism security.

The advantage of this method over conventional fuzzy methods is considering uncertainty, and allocating credit in the opinion of experts to estimate fuzzy parameters.
\end{abstract}

keywords: Z-numbers; Ranking; Centroid Point; Return on assets; Stock exchange

\section{Introduction}

In the real world, to cope with the increasing complexity of research, studies, and modeling and to solve new issues in various sciences, new computational methods were needed.

*Corresponding author. Email address: Mog_afshar@yahoo.com, Tel:+989126776390 
Methods that were more closely related to human thinking. Fuzzy logic is one of them, but Fuzzy logic considers the reliability of the information. To eliminate this restriction Z-number was introduced by Zadeh in 2011 [21].

A Z-number which describes the restriction and the reliability is displayed by an ordered pair of fuzzy numbers $(\bar{A}, \bar{B})$. The first component $\bar{A}$, shows fuzzy restriction and the second component $\bar{B}$, is a reliability of the $\bar{A}[20]$.

As a new concept, discussion about Z-numbers and its basic theories like arithmetic and ranking are few. For solving this problem Kang [11] introduced a method for converting Z-numbers to fuzzy numbers on the base of a fuzzy expectation of a fuzzy set. By this method, the researchers first convert Z-numbers to fuzzy numbers and then by using the theories and the methods of fuzzy numbers solve the problems in the uncertain situations. For example, Bakars and gegov [1] suggested a method that initially turned the Z-number into a fuzzy number then by using the CPS method. This allowed ranking of the generalized fuzzy number.

According to the previous method, it is evident that these methods depend on fuzzy number ranking methods, so before ranking Z-numbers, a new method for ranking generalized fuzzy numbers must be introduced. Chen and Chen [2] introduced a method based on defuzzified values, the highest and the spreads of generalized fuzzy numbers.

As can be seen, converting Z-numbers to generalized fuzzy numbers will lead to the loss of original information [11].

From the last two decades, researchers have given attention to investigating the centroid point of fuzzy numbers and used it for comparing fuzzy numbers. Yager [18] introduced the concept of centroid-based ranking method for comparing fuzzy numbers, but he used only $\mathrm{X}$ coordinate of centroid point. Murakami et al. [14] proposed a centroid-based ranking approach that calculated the centroid point of fuzzy numbers and ranked them according to he larger of X coordinate and Y coordinate. Cheng[3] proposed a ranking method by using distance between original and centroid points. Chu and Tsao [4] proposed a ranking method of fuzzy numbers with an area between the centroid point and the original point. However, Wang et al. [17] demonstrated that the centroid formula defined in [3] and [4] is incorrect and they provided the correct centroid formula. Dat et al. [6] proposed an improved ranking method based on the centroid point for ranking various types of fuzzy numbers. In addition, centroid points have also been analyzed for other generalizations of fuzzy sets $[12,15]$.

In this paper, we have suggested a method that is based on centroid points, and we use them directly for ranking Z-numbers and use this method to rank the return on assets of Tehran stock exchange.

As we know due to the nature of financial markets and the uncertainty in the information available in these markets, it is possible to use Z-numbers to describe future returns in the ranking and selection of investment portfolios.

For example, suppose a capital market expert believes that the return on a particular stock in the next year follows a fuzzy number $A$, but the validity of this prediction can 
be shown by another fuzzy number such as $B$. Therefore, the return of this share can be shown by the number $Z=(\bar{A}, \bar{B})$.

This paper is organized as follows:

Section 2 contains some definitions and concepts of fuzzy numbers and Z-numbers. In section 3 the proposed method for ranking Z-numbers is discussed and then the method is illustrated by a numerical example. In sections 4 there are two practical examples that include ranking of return on assets of stock exchange and ranking of factors affecting the productivity of tourism security by proposed method and interesting and desired results are shown at the end of the section, and the conclusion is presented in section 5 .

\section{Preliminaries}

In this section, we explain some definitions that are used in this paper.

Definition 2.1. A fuzzy subset $\bar{A}=(a, b, c, d ; w)$ of the real line $R$ with membership function $\mu_{\bar{A}}: R \rightarrow[0, w]$, which $0<w<1$, is a fuzzy number if satisfying in the following properties:

1. $\mu_{\bar{A}}$ is a continuous function from $R$ to $[0, w]$.

2. $\mu_{\bar{A}}(x)=0$ for all $x \in(-\infty, a]$ and $x \in[d, \infty)$.

3. $\mu_{\bar{A}}$ is strictly increasing on $[a, b]$.

4. $\mu_{\bar{A}}(x)=w$ for $x \in[b, c]$.

5. $\mu_{\bar{A}}$ is strictly decreasing on $[c, d]$.

If $w=1$ then we say $\bar{A}$ is a normal fuzzy number.

Definition 2.2. A fuzzy number $\bar{A}=(a, b, c, d ; w)$ is said a trapezoidal fuzzy number if it's membership function is as follows:

$$
\mu_{\bar{A}}(x)=\left\{\begin{array}{lr}
0 & x<a \\
\frac{x-a}{b-a} & a \leq x \leq b \\
w & b \leq x \leq c \\
\frac{d-x}{d-c} & c \leq x \leq d \\
0 & x>d
\end{array}\right.
$$

If we have $b=c$ then $\bar{A}$ is said triangular fuzzy number.

As we know, we can approximate each fuzzy number by a trapezoidal number in different approximations. Therefore, in this paper, all of the fuzzy numbers are considered trapezoidal.

Definition 2.3. A Z-number is an ordered pair of fuzzy numbers that is shown as $Z=(\bar{A}, \bar{B})$. The first component $\bar{A}$ is a restriction on the values which $x$ can take and 
the second component $\bar{B}$ is a measure of reliability of the first component, by following membership functions:

$$
\mu_{\bar{A}}(x)=\left\{\begin{array}{lr}
\frac{w_{1}\left(x-a_{1}\right)}{b_{1}-a_{1}} & a_{1} \leq x \leq b_{1} \\
w_{1} & b_{1} \leq x \leq c_{1} \\
\frac{w_{1}\left(d_{1}-x\right)}{d_{1}-c_{1}} & c_{1} \leq x \leq d_{1} \\
0 & \text { o.w }
\end{array}\right.
$$

and

$$
\mu_{\bar{B}}(x)=\left\{\begin{array}{lr}
\frac{w_{2}\left(x-a_{2}\right)}{b_{2}-a_{2}} & a_{2} \leq x \leq b_{2} \\
w_{2} & b_{2} \leq x \leq c_{2} \\
\frac{w_{2}\left(d_{2}-x\right)}{d_{2}-c_{2}} & c_{2} \leq x \leq d_{2} \\
0 & \text { o.w }
\end{array}\right.
$$

where $w_{1}$ and $w_{2}$ are the maximum degree of membership functions.

For $Z=(\bar{A}, \bar{B})$

(1) Convert the $\bar{B}$ (reliability) into crisp number by using

$$
\alpha=\frac{\int_{X} x \mu_{\bar{B}} d x}{\int_{X} \mu_{\bar{B}} d x}
$$

where $\int$ denotes an algebraic integration.

(2) Add the weight of the $\bar{B}$ to the $\bar{A}$ (restriction). The weighted Z-number is denoted as

$$
\bar{Z}^{\alpha}=\left\{\left(x, \mu_{A^{\alpha}}(x)\right) \mid \mu_{A^{\alpha}}(x)=\alpha \mu_{A}(x), x \in[0,1]\right\}
$$

\section{The Proposed Method For Ranking Z-Numbers}

Consider a Z-number as $Z=(\bar{A}, \bar{B})$ so that $\bar{A}=\left(a_{1}, b_{1}, c_{1}, d_{1} ; w_{1}\right)$ and $\bar{B}=\left(a_{2}, b_{2}, c_{2}, d_{2} ; w_{2}\right)$ that their membership functions have been defined in (1) and (2) equations.

Definition3.1. The centroid point of $\bar{A}$ is shown by $\left(x_{\bar{A}}, y_{\bar{A}}\right)$ and is defined as follows:

$$
x_{\bar{A}}=\frac{\int_{a_{1}}^{b_{1}} \frac{x\left(x-a_{1}\right)}{b_{1}-a_{1}} d x+\int_{b_{1}}^{c_{1}} x d x+\int_{c_{1}}^{d_{1}} \frac{x\left(x-d_{1}\right)}{c_{1}-d_{1}} d x}{\int_{a_{1}}^{b_{1}} \frac{\left(x-a_{1}\right)}{b_{1}-a_{1}} d x+\int_{b_{1}}^{c_{1}} d x+\int_{c_{1}}^{d_{1}} \frac{\left(x-d_{1}\right)}{c_{1}-d_{1}} d x} \times w_{1}
$$


and

$$
y_{\bar{A}}=\frac{\int_{0}^{1} y\left(\frac{y\left(b_{1}-a_{1}\right)}{w_{1}}+a_{1}\right) d y+\int_{0}^{1} y\left(\frac{y\left(c_{1}-d_{1}\right)}{w_{1}}+d_{1}\right) d y}{\int_{0}^{1}\left(\frac{y\left(b_{1}-a_{1}\right)}{w_{1}}+a_{1}\right) d y+\int_{0}^{1}\left(\frac{y\left(c_{1}-d_{1}\right)}{w_{1}}+d_{1}\right) d y} \times w_{1}
$$

Similarly the centroid point of $\bar{B}$ as $\left(x_{\bar{B}}, y_{\bar{B}}\right)$ is defined.

Before introducing the algorithm we must note that if the Z-numbers are displayed with $Z_{i}=\left(\bar{A}_{i}, \bar{B}_{i}\right)$ which $i=1,2, \ldots, n$, then it is easily understood that $\bar{A}_{i}$ is the most important part of $Z_{i}$, because the final opportunity of Z-number is to describe the uncertain variable, while $\bar{B}_{i}$, as only a measure of reliability of $\bar{A}_{i}$, can influence the ranking result but can not decide the ranking result, so $\bar{A}_{i}$ is more important and is the main part of Z-number. This point should be considered when we want to rank Z-numbers.

In addition, the information of Z-numbers should be retained as much as we deal with them, so ranking Z-numbers should keep two principles, shown as follows:

(1) $\bar{A}_{i}$ is the main part of Z-number and $\bar{B}_{i}$ is the subsidiary factor of Z-number. So, the weight of $\bar{A}_{i}$ should be larger than $\bar{B}_{i}$ when Z-numbers are ranked.

(2) In the ranking process, the information of Z-number must be kept intact and protected without defects, and the conversion of $\bar{A}_{i}$ and $\bar{B}_{i}$ to a crisp number must be avoided.

So in this algorithm, we try to save most information of Z-numbers by keeping the nature of the Z-numbers.

\section{A Ranking Algorithm Based On Centroid Point}

This section presents a new method that is based on centroid point. Let us consider a set $\Omega$ of $n$ Z-numbers $Z_{i}=\left(\bar{A}_{i}, \bar{B}_{i}\right)$ which $i=1,2, \ldots, n$. We compute the centroid points of $\bar{A}_{i}$ and $\bar{B}_{i}$ then we use their convex combination for definition centroid point of $Z_{i}$, and finally, we rank Z-numbers by using the proposed algorithm. This ranking process can be done according to the following steps:

Step1. For each $Z_{i}=\left(\bar{A}_{i}, \bar{B}_{i}\right)$ such that $\bar{A}_{i}=\left(a_{i 1}, a_{i 2}, a_{i 3}, a_{i 4} ; w_{i \bar{A}}\right)$ and $\bar{B}_{i}=$ $\left(b_{i 1}, b_{i 2}, b_{i 3}, b_{i 4} ; w_{i \bar{B}}\right)$ compute the centroid point for $\bar{A}_{i}$ and $\bar{B}_{i}$ by (3) and (4).

Step2. Suppose that: 


$$
s_{\bar{A}_{i}}=\sqrt{\frac{\sum_{j=1}^{j=4}\left(a_{i j}-\bar{a}_{i j}\right)^{2}}{3}} \quad i=1,2, \ldots, n
$$

and

$$
s_{\bar{B}_{i}}=\sqrt{\frac{\sum_{j=1}^{j=4}\left(b_{i j}-\bar{b}_{i j}\right)^{2}}{3}} \quad i=1,2, \ldots, n
$$

where $\bar{a}_{i j}$ and $\bar{b}_{i j}$ are the mean value of $\bar{A}_{i}$ and $\bar{B}_{i}$ which are defined as follows:

$$
\bar{a}_{i j}=\frac{\sum_{j=1}^{j=4}\left(a_{i j}\right)}{4} \quad i=1,2, \ldots, n
$$

and

$$
\bar{b}_{i j}=\frac{\sum_{j=1}^{j=4}\left(b_{i j}\right)}{4} \quad i=1,2, \ldots, n
$$

Step3. Suppose that $C_{i}=\left(X_{Z_{i}}, Y_{Z_{i}}, S_{Z_{i}}\right)$ such that:

$$
\begin{aligned}
X_{Z_{i}} & =\lambda x_{\bar{A}_{i}}+(1-\lambda) x_{\bar{B}_{i}} \\
Y_{Z_{i}} & =\lambda y_{\bar{A}_{i}}+(1-\lambda) y_{\bar{B}_{i}}
\end{aligned}
$$

and

$$
S_{Z_{i}}=\lambda s_{\bar{A}_{i}}+(1-\lambda) s_{\bar{B}_{i}}
$$

where $\lambda$ is a parameter and $\lambda \in\left[\frac{1}{2}, 1\right]$, and it opposed to $\frac{1}{2}$ if two fuzzy numbers $\bar{A}_{i}$ and $\bar{B}_{i}$ in a Z-number be symmetrical.

Step4. $C^{+}$and $C^{-}$is defined as follows:

$$
C^{+}=\left(X_{\max }, Y_{\max }, S_{\max }\right)
$$

and

$$
C^{-}=\left(X_{\min }, Y_{\min }, S_{\min }\right)
$$

Step5. Evaluation the distance between $C_{i}$ and $C^{+}$for $i=1,2, \ldots, n$ by using Minkowski $L_{P}$ metric as follows:

$$
d\left(C_{i}, C^{+}\right)=\sqrt[p]{\left|X_{\max }-X_{Z_{i}}\right|^{P}+\left|Y_{\max }-Y_{Z_{i}}\right|^{P}+k\left|S_{\max }-S_{Z_{i}}\right|^{P}}
$$

similarly, the distance between $C_{i}$ and $C^{-}$for $i=1,2, \ldots, n$ is measured as follows: 


$$
d\left(C_{i}, C^{-}\right)=\sqrt[p]{\left|X_{\min }-X_{Z_{i}}\right|^{P}+\left|Y_{\min }-Y_{Z_{i}}\right|^{P}+k\left|S_{\text {min }}-S_{Z_{i}}\right|^{P}}
$$

So if the centroid points are equal for two Z-numbers such as $Z_{i}$ and $Z_{j}$, we put $k=1$ and otherwise $k=0$, and $p \geq 1$ is a distance parameter.

Step6. Compute the ranking index that is shown by $R\left(Z_{i}\right)$ as follows:

$$
R\left(Z_{i}\right)=\gamma \frac{\max _{1 \leq k \leq n} d\left(C_{k}, C^{+}\right)-d\left(C_{i}, C^{+}\right)}{\max _{1 \leq k \leq n} d\left(C_{k}, C^{+}\right)-\min _{1 \leq k \leq n} d\left(C_{k}, C^{+}\right)}+(1-\gamma) \frac{d\left(C_{i}, C^{-}\right)-\min _{1 \leq k \leq n} d\left(C_{k}, C^{-}\right)}{\max _{1 \leq k \leq n} d\left(C_{k}, C^{-}\right)-\min _{1 \leq k \leq n} d\left(C_{k}, C^{-}\right)}
$$

Where the parameter $\gamma$ is referred to as the decision maker's preference attitude and $\gamma \in[0,1]$.

Step7. Rank the Z-numbers by using values of $R\left(Z_{i}\right)$ obtained in previous step as follows:

1. We say that $z_{i} \sim z_{j}$ if $R\left(Z_{i}\right)=R\left(Z_{j}\right)$, in the other words if we have:

$$
\begin{gathered}
\gamma \frac{\max _{1 \leq k \leq n} d\left(C_{k}, C^{+}\right)-d\left(C_{i}, C^{+}\right)}{\max _{1 \leq k \leq n} d\left(C_{k}, C^{+}\right)-\min _{1 \leq k \leq n} d\left(C_{k}, C^{+}\right)}+(1-\gamma) \frac{d\left(C_{i}, C^{-}\right)-\min _{1 \leq k \leq n} d\left(C_{k}, C^{-}\right)}{\max _{1 \leq k \leq n} d\left(C_{k}, C^{-}\right)-\min _{1 \leq k \leq n} d\left(C_{k}, C^{-}\right)}= \\
\gamma \frac{\max _{1 \leq k \leq n} d\left(C_{k}, C^{+}\right)-d\left(C_{j}, C^{+}\right)}{\max _{1 \leq k \leq n} d\left(C_{k}, C^{+}\right)-\min _{1 \leq k \leq n} d\left(C_{k}, C^{+}\right)}+(1-\gamma) \frac{d\left(C_{j}, C^{-}\right)-\min _{1 \leq k \leq n} d\left(C_{k}, C^{-}\right)}{\max _{1 \leq k \leq n} d\left(C_{k}, C^{-}\right)-\min _{1 \leq k \leq n} d\left(C_{k}, C^{-}\right)}
\end{gathered}
$$

hence:

$$
\begin{aligned}
& \gamma \frac{\max _{1 \leq k \leq n} d\left(C_{k}, C^{+}\right)-d\left(C_{i}, C^{+}\right)}{\max _{1 \leq k \leq n} d\left(C_{k}, C^{+}\right)-\min _{1 \leq k \leq n} d\left(C_{k}, C^{+}\right)}-\gamma \frac{\max _{1 \leq k \leq n} d\left(C_{k}, C^{+}\right)-d\left(C_{j}, C^{+}\right)}{\max _{1 \leq k \leq n} d\left(C_{k}, C^{+}\right)-\min _{1 \leq k \leq n} d\left(C_{k}, C^{+}\right)}= \\
& (1-\gamma) \frac{d\left(C_{j}, C^{-}\right)-\min _{1 \leq k \leq n} d\left(C_{k}, C^{-}\right)}{\max _{1 \leq k \leq n} d\left(C_{k}, C^{-}\right)-\min _{1 \leq k \leq n} d\left(C_{k}, C^{-}\right)}-(1-\gamma) \frac{d\left(C_{i}, C^{-}\right)-\min _{1 \leq k \leq n} d\left(C_{k}, C^{-}\right)}{\max _{1 \leq k \leq n} d\left(C_{k}, C^{-}\right)-\min _{1 \leq k \leq n} d\left(C_{k}, C^{-}\right)}
\end{aligned}
$$


so we must have:

$$
\gamma \frac{d\left(C_{j}, C^{+}\right)-d\left(C_{i}, C^{+}\right)}{\max _{1 \leq k \leq n} d\left(C_{k}, C^{+}\right)-\min _{1 \leq k \leq n} d\left(C_{k}, C^{+}\right)}=(1-\gamma) \frac{d\left(C_{j}, C^{-}\right)-d\left(C_{i}, C^{-}\right)}{\max _{1 \leq k \leq n} d\left(C_{k}, C^{-}\right)-\min _{1 \leq k \leq n} d\left(C_{k}, C^{-}\right)}
$$

2. We say that $z_{i} \prec z_{j}$ if $R\left(Z_{i}\right)<R\left(Z_{j}\right)$, in the other words if we have:

$$
\begin{gathered}
\gamma \frac{\max _{1 \leq k \leq n} d\left(C_{k}, C^{+}\right)-d\left(C_{i}, C^{+}\right)}{\max _{1 \leq k \leq n} d\left(C_{k}, C^{+}\right)-\min _{1 \leq k \leq n} d\left(C_{k}, C^{+}\right)}+(1-\gamma) \frac{d\left(C_{i}, C^{-}\right)-\min _{1 \leq k \leq n} d\left(C_{k}, C^{-}\right)}{\max _{1 \leq k \leq n} d\left(C_{k}, C^{-}\right)-\min _{1 \leq k \leq n} d\left(C_{k}, C^{-}\right)}< \\
\gamma \frac{\max _{1 \leq k \leq n} d\left(C_{k}, C^{+}\right)-d\left(C_{j}, C^{+}\right)}{\max _{1 \leq k \leq n} d\left(C_{k}, C^{+}\right)-\min _{1 \leq k \leq n} d\left(C_{k}, C^{+}\right)}+(1-\gamma) \frac{d\left(C_{j}, C^{-}\right)-\min _{1 \leq k \leq n} d\left(C_{k}, C^{-}\right)}{\max _{1 \leq k \leq n} d\left(C_{k}, C^{-}\right)-\min _{1 \leq k \leq n} d\left(C_{k}, C^{-}\right)}
\end{gathered}
$$

So we must have:

$$
\gamma \frac{d\left(C_{j}, C^{+}\right)-d\left(C_{i}, C^{+}\right)}{\max _{1 \leq k \leq n} d\left(C_{k}, C^{+}\right)-\min _{1 \leq k \leq n} d\left(C_{k}, C^{+}\right)}<(1-\gamma) \frac{d\left(C_{j}, C^{-}\right)-d\left(C_{i}, C^{-}\right)}{\max _{1 \leq k \leq n} d\left(C_{k}, C^{-}\right)-\min _{1 \leq k \leq n} d\left(C_{k}, C^{-}\right)}
$$

Step8. If one of the component of Z-number be crisp, for example $\bar{A}=(a, a, a, a ; w)$ then we let:

$$
\begin{gathered}
x_{A}=a \\
y_{A}=\frac{w}{2}
\end{gathered}
$$

step9. End.

Theorem 3.1. This algorithm satisfies in following axioms of ranking:

$a_{1}$. For an arbitrary Z-number as $z_{i}$ we have $z_{i} \sim z_{i}$

$a_{2}$. For arbitrary Z-numbers as $z_{i}$ and $z_{j}$ if we have $z_{i} \prec z_{j}$ and $z_{i} \succ z_{j}$ then we should have $z_{i} \sim z_{j}$. 
$a_{3}$. If $z_{i}, z_{j}$ and $z_{k}$ be arbitrary Z-numbers and we have $z_{i} \prec z_{j}$ and $z_{j} \prec z_{k}$ then we have $z_{i} \prec z_{j}$.

$a_{4}$. For three arbitrary Z-numbers $z_{i}, z_{j}$ and $z_{k}$, if we have $z_{i} \prec z_{j}$ then we should have $z_{i}+z_{k} \prec z_{j}+z_{k}$.

$a_{5}$. For arbitrary Z-numbers as $z_{i}$ and $z_{j}$, if $z_{i} \prec z_{j}$ and $k$ be a crisp number such that $k>0$ then we have $k z_{i} \prec k z_{j}$.

\section{proof.}

$a_{1}$. If we put $i=j$ in equation (6), then for an arbitrary Z-number as $z_{i}$ we have:

$$
\gamma \frac{d\left(C_{i}, C^{+}\right)-d\left(C_{i}, C^{+}\right)}{\max _{1 \leq k \leq n} d\left(C_{k}, C^{+}\right)-\min _{1 \leq k \leq n} d\left(C_{k}, C^{+}\right)}=(1-\gamma) \frac{d\left(C_{i}, C^{-}\right)-d\left(C_{i}, C^{-}\right)}{\max _{1 \leq k \leq n} d\left(C_{k}, C^{-}\right)-\min _{1 \leq k \leq n} d\left(C_{k}, C^{-}\right)}
$$

it's clearly that $R\left(Z_{i}\right)=R\left(Z_{i}\right)$ and so $z_{i} \sim z_{i}$.

$a_{2}$. Suppose that $z_{i}$ and $z_{j}$ are two Z-numbers such that $z_{i} \prec z_{j}$ and $z_{i} \succ z_{j}$ so we have:

$$
\gamma \frac{d\left(C_{j}, C^{+}\right)-d\left(C_{i}, C^{+}\right)}{\max _{1 \leq k \leq n} d\left(C_{k}, C^{+}\right)-\min _{1 \leq k \leq n} d\left(C_{k}, C^{+}\right)}<(1-\gamma) \frac{d\left(C_{j}, C^{-}\right)-d\left(C_{i}, C^{-}\right)}{\max _{1 \leq k \leq n} d\left(C_{k}, C^{-}\right)-\min _{1 \leq k \leq n} d\left(C_{k}, C^{-}\right)}
$$

and

$$
\gamma \frac{d\left(C_{j}, C^{+}\right)-d\left(C_{i}, C^{+}\right)}{\max _{1 \leq k \leq n} d\left(C_{k}, C^{+}\right)-\min _{1 \leq k \leq n} d\left(C_{k}, C^{+}\right)}>(1-\gamma) \frac{d\left(C_{j}, C^{-}\right)-d\left(C_{i}, C^{-}\right)}{\max _{1 \leq k \leq n} d\left(C_{k}, C^{-}\right)-\min _{1 \leq k \leq n} d\left(C_{k}, C^{-}\right)}
$$

so:

$$
\gamma \frac{d\left(C_{j}, C^{+}\right)-d\left(C_{i}, C^{+}\right)}{\max _{1 \leq k \leq n} d\left(C_{k}, C^{+}\right)-\min _{1 \leq k \leq n} d\left(C_{k}, C^{+}\right)}=(1-\gamma) \frac{d\left(C_{j}, C^{-}\right)-d\left(C_{i}, C^{-}\right)}{\max _{1 \leq k \leq n} d\left(C_{k}, C^{-}\right)-\min _{1 \leq k \leq n} d\left(C_{k}, C^{-}\right)}
$$

and by (6) we have $z_{i} \sim z_{j}$.

$a_{3}$. Suppose that $z_{i}, z_{j}$ and $z_{k}$ be three Z-numbers and we have $z_{i} \prec z_{j}$ and $z_{j} \prec z_{k}$ so by (5) we should have: 


$$
\begin{gathered}
\gamma \frac{\max _{1 \leq p \leq n} d\left(C_{p}, C^{+}\right)-d\left(C_{i}, C^{+}\right)}{\max _{1 \leq p \leq n} d\left(C_{p}, C^{+}\right)-\min _{1 \leq p \leq n} d\left(C_{p}, C^{+}\right)}+(1-\gamma) \frac{d\left(C_{i}, C^{-}\right)-\min _{1 \leq p \leq n} d\left(C_{p}, C^{-}\right)}{\max _{1 \leq p \leq n} d\left(C_{p}, C^{-}\right)-\min _{1 \leq p \leq n} d\left(C_{p}, C^{-}\right)}< \\
\gamma \frac{\max _{1 \leq p \leq n} d\left(C_{p}, C^{+}\right)-d\left(C_{j}, C^{+}\right)}{\max _{1 \leq p \leq n} d\left(C_{p}, C^{+}\right)-\min _{1 \leq p \leq n} d\left(C_{p}, C^{+}\right)}+(1-\gamma) \frac{d\left(C_{j}, C^{-}\right)-\min _{1 \leq p \leq n} d\left(C_{p}, C^{-}\right)}{\max _{1 \leq p \leq n} d\left(C_{p}, C^{-}\right)-\min _{1 \leq p \leq n} d\left(C_{p}, C^{-}\right)}
\end{gathered}
$$

and

$$
\begin{gathered}
\gamma \frac{\max _{1 \leq i \leq n} d\left(C_{i}, C^{+}\right)-d\left(C_{j}, C^{+}\right)}{\max _{1 \leq p \leq n} d\left(C_{p}, C^{+}\right)-\min _{1 \leq p \leq n} d\left(C_{p}, C^{+}\right)}+(1-\gamma) \frac{d\left(C_{j}, C^{-}\right)-\min _{1 \leq i \leq n} d\left(C_{i}, C^{-}\right)}{\max _{1 \leq p \leq n} d\left(C_{p}, C^{-}\right)-\min _{1 \leq p \leq n} d\left(C_{p}, C^{-}\right)}< \\
\gamma \frac{\max _{1 \leq p \leq n} d\left(C_{p}, C^{+}\right)-d\left(C_{k}, C^{+}\right)}{\max _{1 \leq p \leq n} d\left(C_{p}, C^{+}\right)-\min _{1 \leq p \leq n} d\left(C_{p}, C^{+}\right)}+(1-\gamma) \frac{d\left(C_{k}, C^{-}\right)-\min _{1 \leq p \leq n} d\left(C_{p}, C^{-}\right)}{\max _{1 \leq p \leq n} d\left(C_{p}, C^{-}\right)-\min _{1 \leq p \leq n} d\left(C_{p}, C^{-}\right)}
\end{gathered}
$$

so, we must have:

$$
\begin{gathered}
\gamma \frac{\max _{1 \leq p \leq n} d\left(C_{p}, C^{+}\right)-d\left(C_{i}, C^{+}\right)}{\max _{1 \leq p \leq n} d\left(C_{p}, C^{+}\right)-\min _{1 \leq p \leq n} d\left(C_{p}, C^{+}\right)}+(1-\gamma) \frac{d\left(C_{i}, C^{-}\right)-\min _{1 \leq p \leq n} d\left(C_{p}, C^{-}\right)}{\max _{1 \leq p \leq n} d\left(C_{p}, C^{-}\right)-\min _{1 \leq p \leq n} d\left(C_{p}, C^{-}\right)}< \\
\gamma \frac{\max _{1 \leq p \leq n} d\left(C_{p}, C^{+}\right)-d\left(C_{k}, C^{+}\right)}{\max _{1 \leq p \leq n} d\left(C_{p}, C^{+}\right)-\min _{1 \leq p \leq n} d\left(C_{p}, C^{+}\right)}+(1-\gamma) \frac{d\left(C_{k}, C^{-}\right)-\min _{1 \leq p \leq n} d\left(C_{p}, C^{-}\right)}{\max _{1 \leq p \leq n} d\left(C_{p}, C^{-}\right)-\min _{1 \leq p \leq n} d\left(C_{p}, C^{-}\right)}
\end{gathered}
$$

and then we have $z_{i} \prec z_{k}$.

$a_{4}$. For three Z-numbers $z_{i}, z_{j}$ and $z_{k}$, suppose that $z_{i} \prec z_{j}$, then:

$$
\gamma \frac{\max _{1 \leq p \leq n} d\left(C_{p}, C^{+}\right)-d\left(C_{i}, C^{+}\right)}{\max _{1 \leq p \leq n} d\left(C_{p}, C^{+}\right)-\min _{1 \leq p \leq n} d\left(C_{p}, C^{+}\right)}+(1-\gamma) \frac{d\left(C_{i}, C^{-}\right)-\min _{1 \leq p \leq n} d\left(C_{p}, C^{-}\right)}{\max _{1 \leq p \leq n} d\left(C_{p}, C^{-}\right)-\min _{1 \leq p \leq n} d\left(C_{p}, C^{-}\right)}<
$$




$$
\gamma \frac{\max _{1 \leq p \leq n} d\left(C_{p}, C^{+}\right)-d\left(C_{j}, C^{+}\right)}{\max _{1 \leq p \leq n} d\left(C_{p}, C^{+}\right)-\min _{1 \leq p \leq n} d\left(C_{p}, C^{+}\right)}+(1-\gamma) \frac{d\left(C_{j}, C^{-}\right)-\min _{1 \leq p \leq n} d\left(C_{p}, C^{-}\right)}{\max _{1 \leq p \leq n} d\left(C_{p}, C^{-}\right)-\min _{1 \leq p \leq n} d\left(C_{p}, C^{-}\right)}
$$

and we have:

$$
R\left(z_{k}\right)=\gamma \frac{\max _{1 \leq p \leq n} d\left(C_{p}, C^{+}\right)-d\left(C_{k}, C^{+}\right)}{\max _{1 \leq p \leq n} d\left(C_{p}, C^{+}\right)-\min _{1 \leq p \leq n} d\left(C_{p}, C^{+}\right)}+(1-\gamma) \frac{d\left(C_{k}, C^{-}\right)-\min _{1 \leq p \leq n} d\left(C_{p}, C^{-}\right)}{\max _{1 \leq p \leq n} d\left(C_{p}, C^{-}\right)-\min _{1 \leq p \leq n} d\left(C_{p}, C^{-}\right)}
$$

so, it's clearly that $z_{i}+z_{k} \prec z_{j}+z_{k}$.

$a_{5}$. For arbitrary Z-numbers as $z_{i}$ and $z_{j}$, if $z_{i} \prec z_{j}$ and $k$ be a crisp number such that $k>0$ then we have:

$$
\begin{gathered}
k \gamma \frac{\max _{1 \leq p \leq n} d\left(C_{p}, C^{+}\right)-d\left(C_{i}, C^{+}\right)}{\max _{1 \leq p \leq n} d\left(C_{p}, C^{+}\right)-\min _{1 \leq p \leq n} d\left(C_{p}, C^{+}\right)}+k(1-\gamma) \frac{d\left(C_{i}, C^{-}\right)-\min _{1 \leq p \leq n} d\left(C_{p}, C^{-}\right)}{\max _{1 \leq p \leq n} d\left(C_{p}, C^{-}\right)-\min _{1 \leq p \leq n} d\left(C_{p}, C^{-}\right)}< \\
k \gamma \frac{\max _{1 \leq p \leq n} d\left(C_{p}, C^{+}\right)-d\left(C_{j}, C^{+}\right)}{\max _{1 \leq p \leq n} d\left(C_{p}, C^{+}\right)-\min _{1 \leq p \leq n} d\left(C_{p}, C^{+}\right)}+k(1-\gamma) \frac{d\left(C_{j}, C^{-}\right)-\min _{1 \leq p \leq n} d\left(C_{p}, C^{-}\right)}{\max _{1 \leq p \leq n} d\left(C_{p}, C^{-}\right)-\min _{1 \leq p \leq n} d\left(C_{p}, C^{-}\right)} .
\end{gathered}
$$

and hence $k z_{i} \prec k z_{j}$.

We now use the proven theorems and results from this article in a numerical example and compare the results with the ranking methods found in previous articles.

Example 1. In this example we use 6 sets of Z-numbers to compare ranking results of proposed ranking method for Z-number of existing ranking methods.

For all of the example let $\bar{A}=(0.1,0.3,0.3,0.5 ; 1)$ is the $\bar{A}_{i}$ of $Z_{i}$ in the 6 sets. On the other hands all the Z-numbers have same part $\bar{A}_{i}$. The $\bar{A}$ is shown in Fig.1 and all the $\bar{B}_{i}$ of Z-numbers in the 6 sets are shown in Fig.2. Also in all of the examples we suppose that $\lambda=\frac{1}{2}, p=2$ and $\gamma=\frac{1}{2}$. 


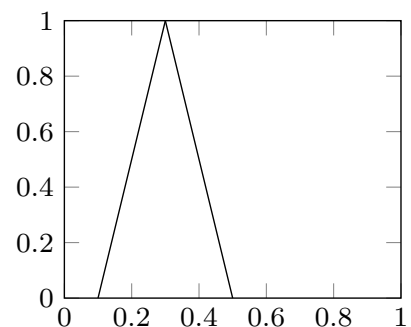

$$
\bar{A}_{i}=(0.1,0.3,0.3,0.5 ; 1)
$$

Fig.1. $\bar{A}_{i}$ of $Z$-numbers in the 6 sets.

set 1

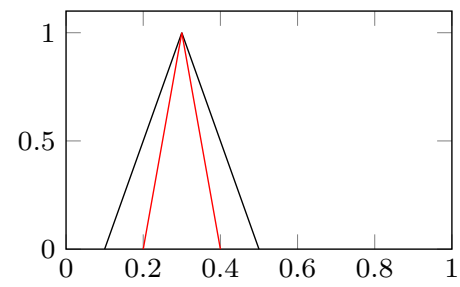

$\bar{B}_{1}=(0.1,0.3,0.3,0.5 ; 1)$

$\bar{B}_{2}=(0.2,0.3,0.3,0.4 ; 1)$

$\operatorname{set} 4$

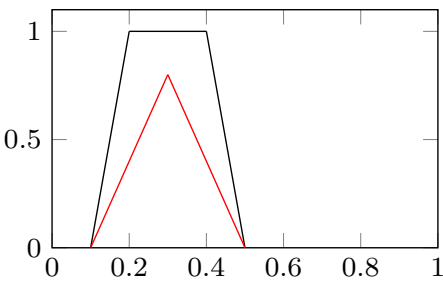

$\overline{B_{1}}=(0.1,0.2,0.4,0.5 ; 1)$

$\bar{B}_{2}=(0.1,0.3,0.3,0.5 ; 0.8)$ set2

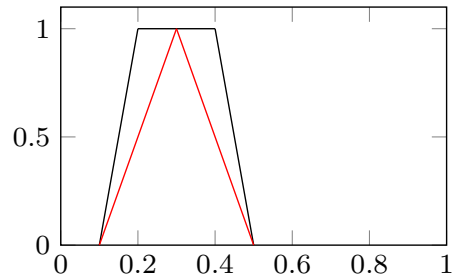

$\overline{B_{1}}=(0.1,0.2,0.4,0.5 ; 1)$

$\overline{B_{2}}=(0.1,0.3,0.3,0.5 ; 1)$

set5

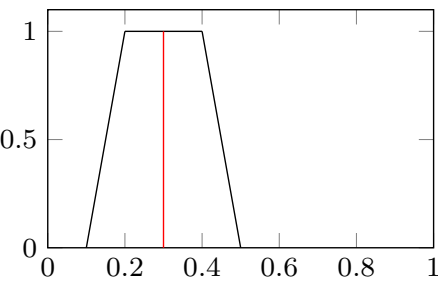

$\overline{B_{1}}=(0.1,0.2,0.4,0.5 ; 1)$

$\overline{B_{2}}=(0.3,0.3,0.3,0.3 ; 1)$ set3

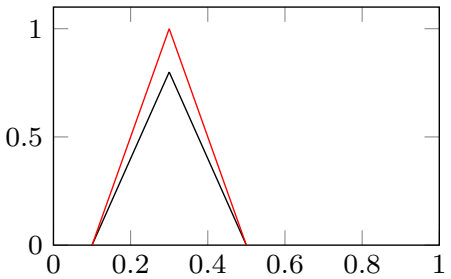

$$
\bar{B}_{1}=(0.1,0.3,0.3,0.5 ; 0.8)
$$$$
\overline{B_{2}}=(0.1,0.3,0.3,0.5 ; 1)
$$

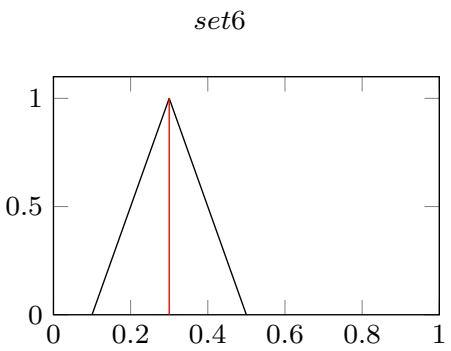

$\overline{B_{1}}=(0.1,0.3,0.3,0.5 ; 1)$

Fig.2. $\bar{B}_{i}$ of $Z-$ numbers in the 6 sets.

In table 1 we have some existed method, and we can see that these methods can not distinguish between Z-numbers. 
Table 1

"A comparison between ranking of previous methods"

\begin{tabular}{|c|c|c|c|c|c|c|}
\hline \multirow[t]{2}{*}{ Methods } & \multicolumn{2}{|c|}{ set1 } & \multicolumn{2}{|c|}{ set2 } & \multicolumn{2}{|c|}{ set3 } \\
\hline & $z_{1}$ & $z_{2}$ & $z_{1}$ & $z_{2}$ & $z_{1}$ & $z_{2}$ \\
\hline Bakar's method [1] & 0.0288 & 0.0288 & 0.0288 & 0.0288 & 0.0288 & 0.0288 \\
\hline Mohammad's method [13] & 0.0774 & 0.0774 & 0.0774 & 0.0774 & 0.0774 & 0.0774 \\
\hline Kang's method [11] & 0.3000 & 0.3000 & 0.3000 & 0.3000 & 0.3000 & 0.3000 \\
\hline The proposed algorithm & 1.0000 & 0.0000 & 1.0000 & 0.0000 & 0.0000 & 1.0000 \\
\hline result of previous methods & \multicolumn{2}{|c|}{$z_{1} \sim z_{2}$} & \multicolumn{2}{|c|}{$z_{1} \sim z_{2}$} & \multicolumn{2}{|c|}{$z_{1} \sim z_{2}$} \\
\hline result of previous methods & \multicolumn{2}{|c|}{$z_{2} \prec z_{1}$} & \multicolumn{2}{|c|}{$z_{2} \prec z_{1}$} & \multicolumn{2}{|c|}{$z_{1} \prec z_{2}$} \\
\hline \multirow[t]{2}{*}{ Methods } & \multicolumn{2}{|c|}{ set4 } & \multicolumn{2}{|c|}{ set5 } & \multicolumn{2}{|c|}{ set6 } \\
\hline & $z_{1}$ & $z_{2}$ & $z_{1}$ & $z_{2}$ & $z_{1}$ & $z_{2}$ \\
\hline Bakar's method [1] & 0.0288 & 0.0288 & 0.0288 & 0.0288 & 0.0288 & 0.0288 \\
\hline Mohammad's method [13] & 0.0774 & 0.0774 & 0.0774 & 0.0774 & 0.0774 & 0.0774 \\
\hline Kang's method [11] & 0.3000 & 0.3000 & 0.3000 & 0.3000 & 0.3000 & 0.3000 \\
\hline The proposed algorithm & 1.0000 & 0.0000 & 0.0000 & 1.0000 & 0.0000 & 1.0000 \\
\hline result of previous methods & \multicolumn{2}{|c|}{$z_{1} \sim z_{2}$} & \multicolumn{2}{|c|}{$z_{1} \sim z_{2}$} & \multicolumn{2}{|c|}{$z_{1} \sim z_{2}$} \\
\hline result of 1 & \multicolumn{2}{|c|}{$z_{2} \prec z_{1}$} & \multicolumn{2}{|c|}{$z_{1} \prec z_{2}$} & \multicolumn{2}{|c|}{$z_{1} \prec z_{2}$} \\
\hline
\end{tabular}

The $\bar{B}_{i}$ 's in the 6 sets are different, but they have a same $\bar{A}_{i}$; hence the different Z-numbers in the 6 sets have same ranking scores by existing methods.

Table 2 clearly shows the desired results of comparing our proposed method with jiang's method [8]. We have ranked 6 different sets by two methods and listed the result.

Table 2

"A comparison between ranking of proposed method and Jiang's method "

\begin{tabular}{|c|c|c|c|c|c|c|}
\hline \multirow{2}{*}{ Method } & \multicolumn{2}{|c|}{ set1 } & \multicolumn{2}{|c|}{ set2 } & \multicolumn{2}{|c|}{ set3 } \\
\hline & $z_{1}$ & $z_{2}$ & $z_{1}$ & $z_{2}$ & $z_{1}$ & $z_{2}$ \\
\hline Jiang's method [8] & 0.1166 & 0.1197 & 0.1217 & 0.1166 & 0.1146 & 0.1166 \\
\hline The proposed algorithm & 1.0000 & 0.0000 & 1.0000 & 0.0000 & 0.0000 & 1.0000 \\
\hline Jiang's result & \multicolumn{2}{|c|}{$z_{1} \prec z_{2}$} & \multicolumn{2}{|c|}{$z_{2} \prec z_{1}$} & \multicolumn{2}{|c|}{$z_{1} \prec z_{2}$} \\
\hline proposed method's result & \multicolumn{2}{|c|}{$z_{2} \prec z_{1}$} & \multicolumn{2}{|c|}{$z_{2} \prec z_{1}$} & \multicolumn{2}{|c|}{$z_{1} \prec z_{2}$} \\
\hline \multirow[t]{2}{*}{ Method } & \multicolumn{2}{|c|}{ set4 } & \multicolumn{2}{|c|}{ set5 } & \multicolumn{2}{|c|}{ set6 } \\
\hline & $z_{1}$ & $z_{2}$ & $z_{1}$ & $z_{2}$ & $z_{1}$ & $z_{2}$ \\
\hline Jiang's method [8] & 0.1217 & 0.1146 & 0.1217 & 0.1512 & 0.1166 & 0.1512 \\
\hline The proposed algorithm & 1.0000 & 0.0000 & 0.0000 & 1.0000 & 0.0000 & 1.0000 \\
\hline Jiang's result & $z_{2}$ & $z_{1}$ & $z_{1}$ & $z_{2}$ & $z_{1}$ & $z_{2}$ \\
\hline proposed method's result & $z_{2}$ & $z_{1}$ & $z_{1}$ & $z_{2}$ & $z_{1}$ & $z_{2}$ \\
\hline
\end{tabular}




\section{Numerical Examples}

\section{Example 4.1. Ranking of Return on Assets of Tehran Stock Exchange}

In this section using the theory of Z-numbers and considering the future return on assets in the form of $\mathrm{z}$ numbers, we will rank the return on assets of Tehran stock exchange. To do this, we have randomly selected ten assets from Tehran stock exchange [9], the symbols of which are listed in Table 2 .

For this purpose, the following three factors are considered.

(1.) Arithmetic mean. Although the arithmetic mean of a return on an asset should not be directly expressed as a future return, it can be considered as a good approximation for its calculation.

(2.) Historical efficiency trend. If recent returns on securities are increasing, it can be believed that the expected return on securities based on historical data is higher than the average, and vice versa. This factor can have a significant impact on estimating future returns on assets.

(3.) Use of financial statements and expert opinion. The use of expert opinion depends on predictions based on financial reports and the personal experience of experts.

The forecast of future return on assets (the first factor of the Z-number) is considered as a trapezoidal number. Also, the validity of these predictions (the second factor of the Z-number) according to the number of fluctuations per share during the last few years as well as the opinion of experts about the probability of predictions, has been considered in the form of symmetrical triangular fuzzy numbers.

For example, suppose an expert believes that the return on a particular share over the next year can be expressed by a trapezoidal fuzzy number in the form of $\bar{A}=(0.12,0.22,0.28,0.35)$. This number indicates that the return on equity will not be less than 0.12 in the next year. On the other hand, the return on this share does not exceed 0.35. Also, the membership rate of share return in the range of 0.22 to 0.28 is equal to 1 . Now, given the person's background, as well as the predictability of the share over the past few years, we can show the confidence level in his prediction equal to another fuzzy triangular number such as $\bar{B}=(0.8,0.9,1)$. In other words, we express the probability that this person's prediction is correct through the fuzzy number $\bar{B}$.

Therefore, the Z-numbers of ten random assets from the Tehran stock exchange are summarized in Table 2. 
"Information about assets and their returns"

\begin{tabular}{ccc}
\hline ROW & Symbol & The Z-number of future returns \\
\hline$A_{1}$ & VNovin & $Z_{1}=((-0.32,0.15,0.30,0.58),(0.6,0.7,0.8))$ \\
\hline$A_{2}$ & Khodro & $Z_{2}=((-0.31,0.15,0.29,0.53),(0.6,0.7,0.8))$ \\
\hline$A_{3}$ & DPars & $Z_{3}=((-0.25,0.12,0.25,0.48),(0.8,0.9,0.1))$ \\
\hline$A_{4}$ & VSandogh & $Z_{4}=((-0.29,0.15,0.31,0.55),(0.7,0.8,0.9))$ \\
\hline$A_{5}$ & VBahman & $Z_{5}=((-0.23,0.11,0.21,0.36),(0.7,0.8,0.9))$ \\
\hline$A_{6}$ & CShoaml & $Z_{6}=((-0.36,0.19,0.37,0.68),(0.8,0.9,1))$ \\
\hline$A_{7}$ & Mellat & $Z_{7}=((-0.26,0.14,0.28,0.50),(0.8,0.9,1))$ \\
\hline$A_{8}$ & RMapna & $Z_{8}=((-0.30,0.17,0.33,0.60),(0.6,0.7,0.8))$ \\
\hline$A_{9}$ & AKhaber & $Z_{9}=((-0.33,0.20,0.35,0.62),(0.7,0.8,0.9))$ \\
\hline$A_{10}$ & BSwich & $Z_{10}=((-0.37,0.21,0.39,0.72),(0.8,0.9,1))$ \\
\hline & &
\end{tabular}

The chart of each Z-number is as follows:

Fig.3.

The Chart Of Factors Return on Assets of Tehran Stock Exchange
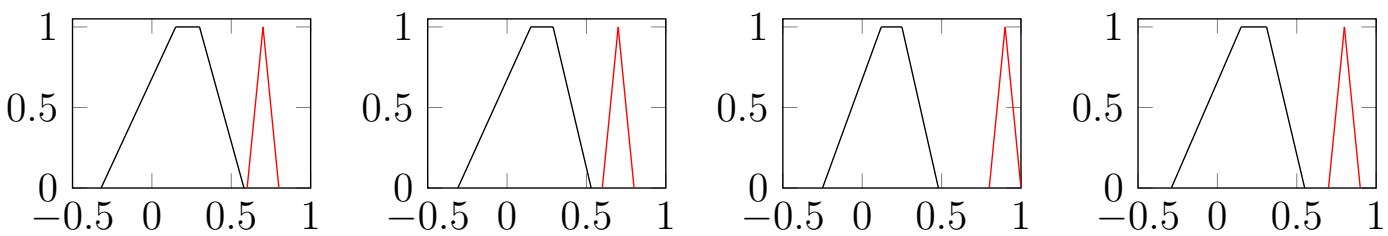

$Z_{1}$

$Z_{2}$

$Z_{3}$

$Z_{4}$
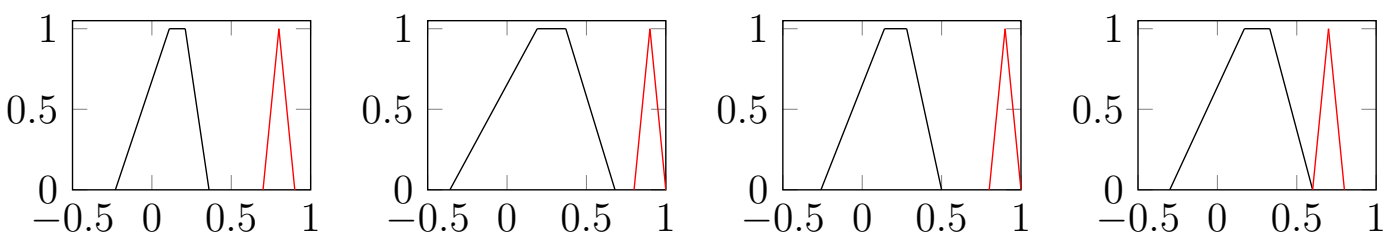

$Z_{5}$

$Z_{6}$

$Z_{7}$

$Z_{8}$
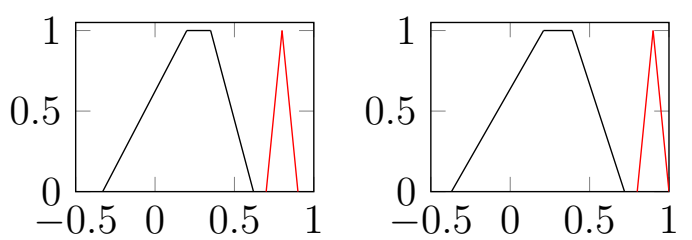

$Z_{9}$

$Z_{10}$ 
Now, by using the proposed algorithm and supposing that $\lambda=\frac{1}{2}, p=2$, we will get that:

Table 4

"The Rank Of Assets"

\begin{tabular}{cccc}
\hline ROW & Symbol & The Z-number of future returns & $R\left(Z_{i}\right)$ \\
\hline$A_{1}$ & VNovin & $Z_{1}=((-0.32,0.15,0.30,0.58),(0.6,0.7,0.8))$ & 0.0313 \\
\hline$A_{2}$ & Khodro & $Z_{2}=((-0.31,0.15,0.29,0.53),(0.6,0.7,0.8))$ & 0 \\
\hline$A_{3}$ & DPars & $Z_{3}=((-0.25,0.12,0.25,0.48),(0.8,0.9,0.1))$ & 0.7325 \\
\hline$A_{4}$ & VSandogh & $Z_{4}=((-0.29,0.15,0.31,0.55),(0.7,0.8,0.9))$ & 0.4468 \\
\hline$A_{5}$ & VBahman & $Z_{5}=((-0.23,0.11,0.21,0.36),(0.7,0.8,0.9))$ & 0.2191 \\
\hline$A_{6}$ & CShoaml & $Z_{6}=((-0.36,0.19,0.37,0.68),(0.8,0.9,1))$ & 0.9620 \\
\hline$A_{7}$ & Mellat & $Z_{7}=((-0.26,0.14,0.28,0.50),(0.8,0.9,1))$ & 0.7923 \\
\hline$A_{8}$ & RMapna & $Z_{8}=((-0.30,0.17,0.33,0.60),(0.6,0.7,0.8))$ & 0.1174 \\
\hline$A_{9}$ & AKhaber & $Z_{9}=((-0.33,0.20,0.35,0.62),(0.7,0.8,0.9))$ & 0.5564 \\
\hline$A_{10}$ & BSwich & $Z_{10}=((-0.37,0.21,0.39,0.72),(0.8,0.9,1))$ & 1.0000 \\
\hline & & &
\end{tabular}

and so we have:

$$
z_{10} \succ z_{6} \succ z_{7} \succ z_{3} \succ z_{9} \succ z_{4} \succ z_{5} \succ z_{8} \succ z_{1} \succ z_{2}
$$

This means that BSwich and CShoaml stocks with the score of 1.000 and 0.9620 have the lowest risk for investment and Khodro stock has the highest risk. So, BSwich is the best choice if you want to invest.

\section{Example 4.2. Ranking of Factors Affecting the Productivity of Tourism Security}

Over the past decades, tourism has continued to grow and diversify and become one of the largest growing economic sectors in the world. Increasing the number of tourists will boost business and increase the income of companies and institutions that operate in this field. The development of tourism is an effective factor in combating poverty and increasing the income of various groups. It reduces unemployment and economic prosperity, and thus improves the quality of peoples lives and increases social welfare.

The tourism industry today is more diverse and complex than in the past. At present, tourists have shorter stays in tourist destinations. Instead of long stays, they have increased the number of their trips. They place more emphasis on the destination environment, expect more value for money, and demand better services. As a result, in order to 
succeed in tourism, a region must be economically, socially and environmentally secure, and in order to be secure, it must be carefully planned managed, and taken various factors into consideration. In fact, improving security in tourist areas is an attractive key factor in encouraging tourists to travel safely. It can be an incentive for people and industries to provide services and facilities for the development of this industry.

In this section, we prioritize the factors affecting the productivity of tourism security and Isfahan (in Iran) has been selected as a case study. Based on the research background, a series of studies conducted on sustainable tourism security in Iran, as well as the views of professors and experts at various levels in the field of tourism in Isfahan, 15 factors affecting security efficiency in tourism supply, including attraction, transportation, Information, advertising, and services were identified in the form of a questionnaire.[7]

To rank the factors, 15 factors were selected as follows:

$A_{1}$. Cooperation of travel agencies and tour guides with the security police.

$A_{2}$. Cooperation of hotel officials with security police.

$A_{3}$. Establishment of parking lots in and out of tourist places for security and environmental protection.

$A_{4}$. Using of public tourist transport fleet equipped with GPS and approved by the police. $A_{5}$. Modification of roads and installation of traffic signs on tourist routes.

$A_{6}$. Using a comprehensive database to obtain visas and tourist information.

$A_{7}$. Creating a comprehensive security control system, intelligent law enforcement to know the status of tourists.

$A_{8}$. Developing virtual tourism to combat malicious advertising.

$A_{9}$. Developing ethical standards for tourism police personnel in dealing with tourists. $A_{10}$. Informing tourists of local values and laws and the need to respect these values. $A_{11}$. Providing timely services for renewal and obtaining visas and accommodation for foreign tourists.

$A_{12}$. The seriousness of the police in pursuing the complaints of tourists.

$A_{13}$. Strengthening the expert force in all areas of tourism.

$A_{14}$. Existence of 24-hour police patrols in seasons when there are many tourists.

$A_{15}$.Periodic police visits of railways, bus terminals, hotels, and camps. 
Table 5

"Information about 15 Factors affecting security and tourism productivity"

\begin{tabular}{cc}
\hline ROW & The Z-number of affecting security and tourism productivity \\
\hline$A_{1}$ & $Z_{1}=((0.457,0.502,0.523),(0.5,0.6,0.7))$ \\
\hline$A_{2}$ & $Z_{2}=((0.428,0.769,0.771),(0.6,0.7,0.8))$ \\
\hline$A_{3}$ & $Z_{3}=((0.465,0.729,0.734),(0.7,0.8,0.9))$ \\
\hline$A_{4}$ & $Z_{4}=((0.378,0.813,0.820),(0.8,0.9,1))$ \\
\hline$A_{5}$ & $Z_{5}=((0.375,0.825,0.830),(0.7,0.8,0.9))$ \\
\hline$A_{6}$ & $Z_{6}=((0.369,0.831,0.859),(0.8,0.9,1))$ \\
\hline$A_{7}$ & $Z_{7}=((0.339,0.851,0.859),(0.7,0.8,0.9))$ \\
\hline$A_{8}$ & $Z_{8}=((0.352,0.839,0.852),(0.7,0.8,0.9))$ \\
\hline$A_{9}$ & $Z_{9}=((0.377,0.722,0.725),(0.6,0.7,0.8))$ \\
\hline$A_{10}$ & $Z_{10}=((0.404,0.553,0.578),(0.7,0.8,0.9))$ \\
\hline$A_{11}$ & $Z_{11}=((0.355,0.840,0.857),(0.8,0.9,1))$ \\
\hline$A_{12}$ & $Z_{12}=((0.377,0.812,0.821),(0.7,0.8,0.9))$ \\
\hline$A_{13}$ & $Z_{13}=((0.376,0.814,0.823,0.72),(0.8,0.9,1))$ \\
\hline$A_{14}$ & $Z_{14}=((0.355,0.846,0.864),(0.8,0.9,1))$ \\
\hline$A_{15}$ & $Z_{15}=((0.383,0.810,0.814),(0.7,0.8,0.9))$ \\
\hline
\end{tabular}

The chart of Z-numbers are as follows:

Fig.4.

The Chart Of Factors Affacting Security And Tourism Productivity

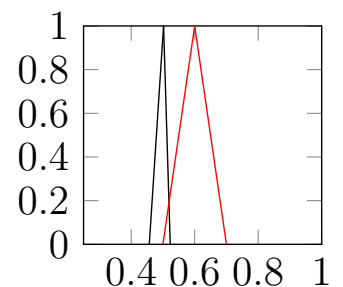

$Z_{1}$

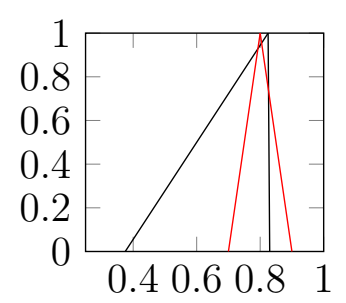

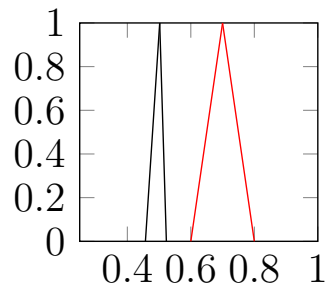

$Z_{2}$

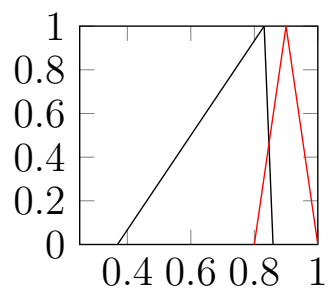

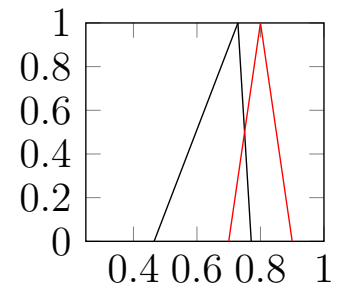

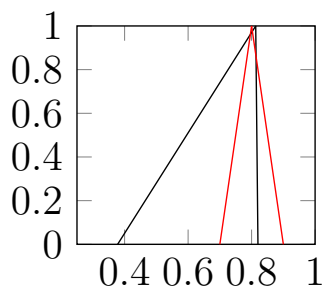

$Z_{3}$

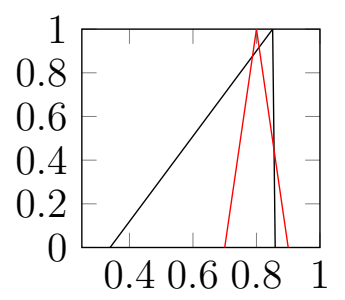

$Z_{4}$

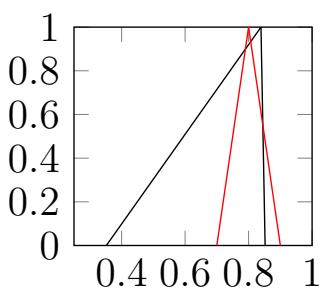


$Z_{5}$

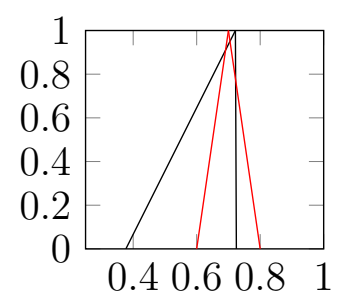

$Z_{6}$

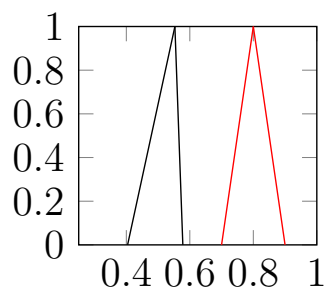

$Z_{7}$

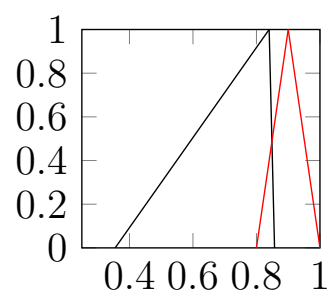

$Z_{8}$

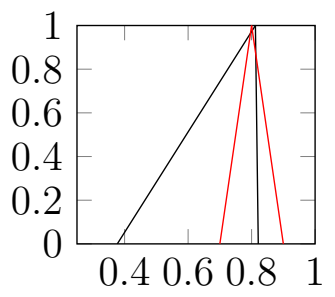

$Z_{9}$

$Z_{10}$

$Z_{11}$

$Z_{12}$

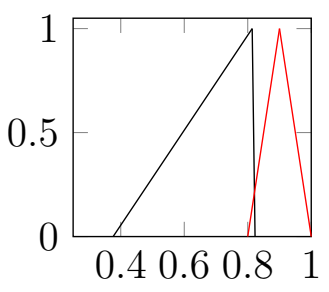

$Z_{13}$

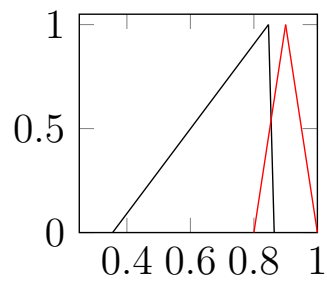

$Z_{14}$

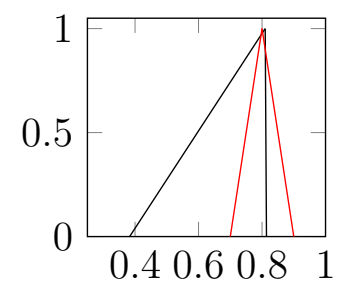

$Z_{15}$

Now using the proposed algorithm, we have the following ranking table (6):

Table 6

"Ranked of the Factors affecting security and tourism productivity"

\begin{tabular}{ccc}
\hline ROW & The Z-number of affecting security and tourism productivity & Score of $Z_{i}$ \\
\hline$A_{1}$ & $Z_{1}=((0.457,0.502,0.523),(0.5,0.6,0.7))$ & 0.0000 \\
\hline$A_{2}$ & $Z_{2}=((0.428,0.769,0.771),(0.6,0.7,0.8))$ & 0.6182 \\
\hline$A_{3}$ & $Z_{3}=((0.465,0.729,0.734),(0.7,0.8,0.9))$ & 0.7218 \\
\hline$A_{4}$ & $Z_{4}=((0.378,0.813,0.820),(0.8,0.9,1))$ & 0.9493 \\
\hline$A_{5}$ & $Z_{5}=((0.375,0.825,0.830),(0.7,0.8,0.9))$ & 0.8244 \\
\hline$A_{6}$ & $Z_{6}=((0.369,0.831,0.859),(0.8,0.9,1))$ & 0.9965 \\
\hline$A_{7}$ & $Z_{7}=((0.339,0.851,0.859),(0.7,0.8,0.9))$ & 0.8549 \\
\hline$A_{8}$ & $Z_{8}=((0.352,0.839,0.852),(0.7,0.8,0.9))$ & 0.8490 \\
\hline$A_{9}$ & $Z_{9}=((0.377,0.722,0.725),(0.6,0.7,0.8))$ & 0.4796 \\
\hline$A_{10}$ & $Z_{10}=((0.404,0.553,0.578),(0.7,0.8,0.9))$ & 0.3424 \\
\hline$A_{11}$ & $Z_{11}=((0.355,0.840,0.857),(0.8,0.9,1))$ & 0.9899 \\
\hline$A_{12}$ & $Z_{12}=((0.377,0.812,0.821),(0.7,0.8,0.9))$ & 0.8051 \\
\hline$A_{13}$ & $Z_{13}=((0.376,0.814,0.823,0.72),(0.8,0.9,1))$ & 0.9533 \\
\hline$A_{14}$ & $Z_{14}=((0.355,0.846,0.864),(0.8,0.9,1))$ & 1.0000 \\
\hline$A_{15}$ & $Z_{15}=((0.383,0.810,0.814),(0.7,0.8,0.9))$ & 0.8007 \\
\hline
\end{tabular}


So we have:

$Z_{1} \prec Z_{10} \prec Z_{9} \prec Z_{2} \prec Z_{3} \prec Z_{15} \prec Z_{12} \prec Z_{5} \prec Z_{8} \prec Z_{7} \prec Z_{4} \prec Z_{13} \prec Z_{11} \prec Z_{6} \prec$
$Z_{14} \cdot$

It means that the presence of 24-hour police patrols in seasons when there are many tourists, can have the greatest impact on the security and productivity of tourism. Therefore, in allocating the tourism budget, more attention should be paid to this part and more manpower should be considered for it.

And the creating a comprehensive security control system, intelligent law enforcement to know the status of tourists will have less impact, so the lowest budget belongs to this item.

\section{Conclusion}

In this paper, we presented a new method for ranking Z-numbers. In comparison with the existing methods for ranking Z-numbers, the proposed method has the advantage that it does not convert $z_{i}$ to fuzzy number. We considered a Z-number as $z=(\bar{A}, \bar{B})$, then we obtained the centroid points of $\bar{A}$ and $\bar{B}$, and with a new ranking algorithm, based on calculation the distance between centroid points, ranked Z-numbers.

Finally, two numerical examples for this ranking method were presented. First, by selecting several assets from the Tehran Stock Exchange, the ranking of these assets was done. As it has been shown, the use of Z-numbers in the problem of investment ranking causes that in addition to allocating uncertainty on the return on assets, the amount of credit for the forecast of experts is also taken into account. In fact, using Z-numbers theory, uncertainty in estimating fuzzy parameters can also be considered.

Then, in the second example, we used a descriptive method to rank the factors affecting the productivity of tourism security and tried to use the knowledge of experts and specialists in this industry to rank the indicators for evaluating tourist attractions.

\section{Declarations}

Conflict of Interest

The authors declare that they have no conflict of interest regarding the publication of this paper.

Ethical Approval

This article does not contain any studies with human participants or animals performed by any of the authors.

\section{Data Availability}


The datasets generated and analysed during the current study are available from the corresponding author on reasonable request.

\section{Authorship Contributions}

M. Afshar kermani and T. Allahviranloo devised the project, the main conceptual ideas and proof outline and verified the analytical methods. M. Farzam developed the theory and performed the computations and wrote the manuscript with support from M. Afshar kermani and T. Allahviranloo. M. Afshar kermani encouraged M. Farzam to investigate and supervised the findings of this work. All authors discussed the results and contributed to the final manuscript.

\section{References}

[1] A. S. A. Bakar, A. Gegov, Multi-layer decision methodology for ranking Z-numbers, International Journal of Computational Intelligence Systems 8 (2015) 395406.

[2] S.M. Chen, A. Munif, G.-S. Chen, H.-C. Liu, B.C. Kuo, Fuzzy risk analysis based on ranking generalized fuzzy numbers with different left heights and right heights, Expert Systems With Applications 39(2012)63206334.

[3] C.H. Cheng. A new approach for ranking fuzzy numbers by distance method, Fuzzy Sets and Systems 95(3)(1998) 307-317.

[4] T. C. Chu ,C. T. Tsao, Ranking fuzzy numbers with an area between the centroid point and original point, Computers and Mathematics with Applications 43(1)(2002)111 - 117. [5] S. Das, D. Guha, A centroid-based ranking method of trapezoidal intuitionistic fuzzy numbers and its application to MCDM problems, Fuzzy Information and Engineering, $8(2016) 41-74$.

[6] L. Q. Dat, V. F. Yu, S. Y. Chou, An improved ranking method for fuzzy numbers based on the centroid index, International Journal of Fuzzy Systems 14(3)(2012)413 - 419.

[7] M. H. Haashemi, Identifying and Prioritizing Effective Factors on the Tourism Security Productivity and Representing functional model (Case Study: Isfahan, Iran), Isfahan Law Enforcement Quarterly, (2015) $61-90(6)$.

[8] W. Jiang, C. Xie, Y. Luo, Y. Tang, Ranking Z-numbers with an improved ranking method for generalized fuzzy numbers. Journal of Intelligent and Fuzzy Systems, Accepted (2016).

[9] A.A. jirofti, A.A. najafi, Optimizing the investment portfolio by risk-based value under credit theory with Z-numbers approach, FEJ, Volume 8, Issue 30, Pages 95 - 113, (2017). [10] B. Kang, D. Wei, Y. Li, Y. Deng, A method of converting Z-number to classical fuzzy number, Journal of Information and Computational Science 3 (2012) 703709 .

[11] B. Kang, D. Wei, Y. Li, Y. Deng, Decision making using Z-numbers under uncertain environment, Journal of Computational Information Systems 8(2012), 2807-2814.

[12] N.N. Karnik, J.M. Mendel, Centroid of a type-2 fuuzzy set, Information Sciences 
132(2001) 195-220.

[13] D. Mohamad, S.A. shahrani and N.H. Kamis, A Z-number based decision making procedure with ranking fuzzy numbers method, AIP Conference Proceedings 1653(2014), 160-166.

[14] S. Murakami, H. maeda, S. Imamura, Fuzzy decision analysis in the development of centralized regional energy control systems, Energy Developments in Japan 6(4)(1984)379396.

[15] J.T. Starczewski, Centroid of triangular and Gaussian type-2 fuzzy sets, Information sciences 280(2014) 289-306.

[16] X. Wang , E. Kerre , Reasonable properties for the ordering of fuzzy quantities (I) , Fuzzy sets and systems ,118(2001)375 - 385 .

[17] Y. M.Wang, J. B. Yang, D. L. Xu, K. S. Chin, On the centroids of fuzzy numbers, Fuzzy Sets and Systems 157(2006)919926.

[18] R.R. Yager, On a general class of fuzzy connectives, Fuzzy Sets and Systems 4(3) (1980) 235-242.

[19] R.R. Yager, On a view of Zadeh's Z-numbers, Springer-Verlag Berlin Heidelberg, (2012a)90-101.

[20] R.R. Yager, On Z-valuations using Zadeh's Z-numbers, International Journal of Intelligent Systems, 27(3)(2012b)259-278.

[21] L. A. Zadeh, A note on Z-numbers, Information Sciences, 181 (2011), 2923 - 2932. 